\title{
THE STRUCTURE OF NESTED SPACES
}

\section{BY}

\author{
T. B. MUENZENBERGER AND R. E. SMITHSON
}

\begin{abstract}
The structure of nested spaces is studied in this paper using such tools as branches, chains, partial orders, and rays in the context of semitrees. A classification scheme for various kinds of acyclic spaces is delineated in terms of semitrees. Several families of order compatible topologies for semitrees are investigated, and these families are grouped in a spectrum (inclusion chain) of topologies compatible with the semitree structure. The chain, interval, and tree topologies are scrutinized in some detail. Several topological characterizations of semitrees with certain order compatible topologies are also derived.
\end{abstract}

1. Introduction. Certain specialized types of acyclic spaces have been studied extensively by many authors. Whyburn examined dendrites in some detail [31], and Plunkett later characterized dendrites in terms of the fixed point property [19]. Ward investigated trees in a series of papers ([24], [25]), and he in turn characterized dendroids in terms of the fixed point property [29]. Ward also introduced several generalizations of trees which included arboroids [27] and generalized trees [25]. Charatonik and Eberhart have recently published papers on dendroids and fans ([4], [5], [6]). In 1946 Young defined nested spaces [34], and he continued the research in a later paper [35]. Borsuk considered [3] a special type of nested space that Holsztyński later termed a $B$ space [11]. When in 1972 Bellamy proved that any arcwise connected continuum is decomposable [2], it became clear that all of the aforementioned acyclic continua are nested, and this fact justifies a thorough investigation of nested spaces. The purpose of the present paper is to initiate a study of the algebraic and topological structure of nested spaces in the spirit of Wallace and Ward ([24], [25], [27]).

A semitree is simply the extracted algebraic structure of a nested space. Many known theorems for trees can be extended to semitrees that possess an

Presented in part to the Society, January 28, 1973 under the title The structure of semitrees. I; received by the editors August 15, 1973.

AMS (MOS) subject classifications (1970). Primary 54F05.

Key words and phrases. Acyclic spaces, arboroids, arcs, box topology, branches, chain topology, combs, fans, nested spaces, order compatible topologies, partially ordered spaces, semitrees, trees. 
order compatible topology in the sense of Wolk [32], and many new results can be established. Thus compactness, local connectivity, and metrizability are in no way essential. The primary application of semitrees at the present time has been to provide a structure sufficient to prove the classical fixed point theorems for multifunctions on acyclic spaces ([15], [17]).

The algebraic properties of semitrees are reviewed in $\S 2$, and rays and branches are considered in $\S 3$. Semitrees possessing certain types of order compatible topologies are studied in $\S 4$. Many examples are presented in $\S 5$, and chains are characterized in $\S 6$. Finally, three topological characterizations of semitrees are established in $\$ 7$.

2. Algebraic semitrees. The fundamental facts about the algebraic structure of semitrees are reviewed in this section. Nearly all of the following results were proved in [15]. The basic structural requirements on a semitree are very simple. A semitree must have unique arcs and contain no open rays. Formally, a semitree consists of a pair $(X, P)$ where $X$ is a nonempty set and $P$ is a family of subsets of $X$ together with six axioms on the pair $(X, P)$.

Aхıом 1. For all $x, y \in X$ there exists a unique minimal (under inclusion) element of $P$ containing $x$ and $y$.

The minimal element of $P$ that contains $x$ and $y$ is called the chain in $X$ with endpoints $x$ and $y$ and is denoted by $[x, y]$. Define in the usual way $(x, y)=[x, y] \backslash\{x, y\}$ and $(x, y]=[x, y] \backslash\{x\}=[y, x)$.

Axiom 2. If $\varnothing \neq P_{0} \subset P$ and if $\cap P_{0} \neq \varnothing$, then $\cap P_{0} \in P$.

Aхіом 3. For all $P \in P$ there exists a unique pair $x, y \in X$ such that $P=[x, y]$.

Thus $P$ consists of the chains in $X$. A set $A \subset X$ is chainable if and only if for all $x, y \in A$ it follows that $[x, y] \subset A$.

Axiom 4. The union of two chains with nonempty intersection is chainable.

Axiom 5. If $P_{0} \subset P$ is a nest, then there exists $P \in P$ such that $\cup P_{0} \subset P$.

Ахіом 6. If $x, y \in X$ and if $x \neq y$, then $(x, y) \neq \varnothing$.

A pair $(X, P)$ satisfying Axioms $1-6$ is called a semitree. If, in addition, $X$ has some topological structure, then the pair $(X, P)$ will occasionally be referred to as an algebraic semitree.

Pick a basepoint $e \in X$ and define a relation $\leqslant$ by the rule: $x \leqslant y$ if 
and only if $x \in[e, y]$. If $x \leqslant y$ and $x \neq y$, then write $x<y$. The relation $\leqslant$ is called the semitree order with basepoint $e$. For $x \in X$ define

$$
L(x)=\{y \in X \mid y \leqslant x\} \text { and } M(x)=\{y \in X \mid x \leqslant y\} .
$$

When more than one basepoint is being considered, the notations $\leqslant_{e}, L_{e}(x)$, and $M_{e}(x)$ will be used to indicate the dependence on the basepoint. If $x \leqslant y$, then $[x, y]$ is called an ordered chain. If $x$ and $y$ are not comparable under $\leqslant$, then $[x, y]$ is called an unordered chain. A triple $(X, P, \lessgtr)$ will occasionally be called a semitree in order to stress a particular order $\leqslant$.

In the following six lemmas, $(X, P, \leqslant)$ is assumed to be a semitree where $\leqslant$ is a specific semitree order with basepoint $e$.

LEMMA 2.1. (i) For all $x, y \in X, x \leqslant y$ if and only if $[e, x] \subset[e, y]$.

(ii) The semitree order $\leqslant_{e}$ is an order dense partial order with unique least element $e$.

(iii) The set $X$ is chainable and does not contain two distinct chains with the same endpoints.

(iv) An intersection of chainable sets is chainable.

Lemma 2.2. Suppose that $x, y, z \in X$ where $x \leqslant y \leqslant z$. Then

(i) $[x, x]=\{x\}=L(x) \cap[x, y]$;

(ii) $L(y)=[e, y]=L(x) \cup[x, y]$;

(iii) $L(y) \backslash L(x)=(x, y]$;

(iv) $[x, y]=L(y) \cap M(x)$;

(v) $[x, z]=[x, y] \cup[y, z]$.

Lemma 2.3. Suppose that $x, y \in X$ where $x \neq y$. Then

(i) $[x, y],[x, y),(x, y]$, and $(x, y)$ are chainable;

(ii) if $z \in(x, y)$, then $[x, y] \backslash\{z\}$ is not chainable;

(iii) $L(x)$ is totally ordered by $\leqslant$;

(iv) $M(x), X \backslash M(x)$, and $X \backslash(M(x) \backslash\{x\})$ are all chainable.

LEMMA 2.4. (i) The pair $(X, \leqslant)$ is a complete semilattice directed downward, and if $\varnothing \neq A \subset[x, y]$, then $\inf A \in[x, y]$.

(ii) If $\varnothing \neq A \subset X$ is totally ordered, then $A$ has a supremum in $X$; if moreover, $A \subset[x, y]$, then $\sup A \in[x, y]$.

(iii) If $x \leqslant y$, then $[x, y]$ is a complete lattice.

The notation $x \wedge y$ will be used for the point inf $\{x, y\}=\sup (L(x) \cap L(y))$ where $x, y \in X$. When $x$ and $y$ are comparable, the notation $x \vee y$ will stand for the point $\sup \{x, y\}$. 
Lemma 2.5. Suppose that $x, y \in X$. Then

(i) $L(x) \cap L(y)=L(x \wedge y)$;

(ii) $[x, y]=[x \wedge y, x] \cup[x \wedge y, y]$;

(iii) $[x \wedge y, x] \cap[x \wedge y, y]=\{x \wedge y\}$;

(iv) if $x$ and $y$ are not comparable, then no $p \in(x \wedge y, x]$ is comparable to any $q \in(x \wedge y, y]$.

LEMMA 2.6. If $A$ is chainable and if $A$ meets both $M(x)$ and $X \backslash M(x)$, then $x \in A$.

It is possible to give an order theoretic characterization of semitrees. Let $(X, \preccurlyeq)$ be a partially ordered set satisfying the following five conditions:

(a) There is a least element $e \in X$;

(b) the partial order $\leqslant$ is order dense;

(c) if $\varnothing \neq A \subset X$, then $\inf A$ exists in $X$;

(d) every nonempty totally ordered subset of $X$ has a supremum in $X$;

(e) for each $x \in X, L(x)$ is totally ordered.

Define the chains in $X$ as follows. If $x \leqslant y$, then set $[x, y]=$ $\{z \in X \mid x \leqslant z \leqslant y\}$. If $x$ and $y$ are not comparable, then set $[x, y]=$ $[x \wedge y, x] \cup[x \wedge y, y]$. Let $P$ be the collection of all sets $[x, y]$ for $x, y \in X$.

THEOREM 2.7. The pair $(X, P)$ is a semitree, and the semitree order $\leqslant_{e}$ is precisely the original order $\leqslant$. Conversely, any semitree $(X, P)$ yields a partially ordered set $(X, \leqslant)$ satisfying conditions (a)-(e) above where $\leqslant$ is any semitree order.

3. Rays and branches. Certain maximal subsets of semitrees have proved extremely useful in two other papers ([15], [16]), and they serve to elucidate the algebraic structure of a semitree. Let $(X, P, \leqslant)$ be a semitree and let $x \in X$. A ray at $x$ is a union of a maximal nest of chains in $M(x)$ which have $x$ as a common endpoint. A major part of the following theorem was proved in [15]. The significance of Axiom 5 of $\$ 2$ lies in proving the existence of maximal elements.

THEOREM 3.1. In a semitree $(X, P, \leqslant)$ the following hold:

(i) Every ray at $x$ is a chain with one endpoint being $x$;

(ii) for each $x \in X$ there exists a maximal element $m \in X$ such that $x \leqslant m$;

(iii) an element $m$ of $X$ is maximal if and only if $[x, m]$ is a ray at $x$ for each $x \in X$ with $x \leqslant m$;

(iv) a subset $A$ of $X$ is a maximal totally ordered subset of $X$ if and only if $A$ is a ray at $e$; 
(v) the semitree $X$ has the representation $X=\bigcup\{[e, m] \mid m$ is a maximal element in $X\}$.

Let $x \in X$. A branch $B$ at $x$ is a subset of $M(x)$ which is maximal with respect to the property: If $y, z \in B \backslash\{x\}$, then $y \wedge z \in B \backslash\{x\}$. This definition could be made in an arbitrary semilattice $(X, \wedge)$. Notice that the definition of brancin depends on a particular semitree order $\leqslant_{e}$. Also there is one other candidate for a branch at $x$; namely, $X \backslash(M(x) \backslash\{x\})$. In fact, this set would be a branch at $x$ if the semitree order $\xi_{x}$ were used. For the purposes of this paper, branches at $x$ will be subsets of $M_{e}(x)$. Standard maximality arguments establish the next two lemmas and corollary which contain the elementary algebra of branches. Most of the following results are valid in a more general setting.

LemMA 3.2. Let $B$ be a branch at $x$. Then

(i) $x \in B$ and $B$ is chainable;

(ii) if $y \in B \backslash\{x\}$, then $M(y) \subset B$;

(iii) every $y \in M(x)$ is contained in a branch at $x$;

(iv) any two distinct branches at $x$ meet only at $x$, and thus there is exactly one branch at $x$ containing $y \in M(x) \backslash\{x\}$;

(v) $B=\bigcup\{M(y) \mid y \in B \backslash\{x\}\} \cup\{x\}$ and $B \backslash\{x\}=\bigcup\{M(y) \backslash\{y\} \mid y \in$ $B \backslash\{x\}\}$.

COROLlary 3.3. The set $M(x)$ is the union of all branches at $x$. If $x \leqslant y$, then $M(x)=[x, y] \cup M(y) \cup \cup\{B \mid B$ is a branch at $z \in[x, y)$ and $B \cap[x, y]=\{z\}\}$.

LEMMA 3.4. Let $B_{x}$ and $B_{y}$ be branches at $x$ and $y$, respectively. Then the following hold:

(i) If $x \notin B_{y}$ and $y \notin B_{x}$, then $B_{x} \cap B_{y}=\varnothing$;

(ii) if $x \leqslant y, B_{x} \neq B_{y}, a \in B_{x}, b \in B_{y}$, and $B_{x} \cap[x, y]=\{x\}$, then $a \wedge b=x$.

The following theorem contains an equivalent formulation of branches in terms of rays in a semitree.

THEOREM 3.5. Let $B$ be a branch at $x$. Then

(i) There exists a maximal element $m \in X$ such that $m \in B$;

(ii) if $m \in B$ is a maximal element, then $B=[x, m] \cup \cup\{R \mid R$ is a ray at $y \in(x, m]\}$.

Proof. The first part of the theorem follows easily from Theorem 3.1(ii) and Lemma 3.2(ii). Let $m \in B$ be a maximal element. Clearly, the right-hand side of the above equation is contained in $B$ by Lemma 3.2(i) and (ii). 
Suppose that $p \in B \backslash[x, m]$. Then $m, p \in B \backslash\{x\}$, and so $y=m \wedge p \in B \backslash\{x\}$. Obviously, $p$ is an element of some ray at $y \in(x, m]$ by Theorem 3.1.

4. Topological semitrees. Five kinds of natural topologies for a semitree are considered in this section. Many folk theorems for trees are then extended to semitrees, and some results of a new and different character are derived. We examine the relationships between the topologies and indicate in $\S 5$ that a semitree can have infinitely many natural topologies, not all of which are related. Let $\left(X, P, \leqslant_{e}\right)$ be an algebraic semitree throughout this section.

Let $A$ be a nonempty subset of $X$. A point $x \in X$ is a quasi supremum of $A$ if and only if

(a) for all $a \in A, x \nless a$ and

(b) for all $y \in X$ such that $y \nless a$ for each $a \in A, y \nless x$.

The set $A$ is closed above if and only if $A$ contains all of its quasi suprema. The set $A$ is closed below if and only if $\inf A \in A$.

A set $A \subset X$ is closed if and only if for all $x, y \in X$ with $x \leqslant y$, $\inf (A \cap[x, y]) \in A$ and $\sup (A \cap[x, y]) \in A$ whenever $A \cap[x, y] \neq \varnothing$. Equivalently, $A \subset X$ is closed if and only if the infimum and supremum of each nonempty totally ordered subset of $A$ are back in $A$.

The next theorem says that a quasi supremum of a chainable set is the supremum of a part of the set.

THEOREM 4.1. If $\varnothing \neq A \subset X$ is chainable, then $x \in X$ is a quasi supremum of $A$ if and only if $A \cap[e, x] \neq \varnothing$ and $x=\sup A \cap[e, m]$ for each $m \geqslant x$.

Proof. Suppose first that $x \in X$ is a quasi supremum of $A$. If $x \in A$, then there is nothing to prove. So choose $a \in A \backslash\{x\}$. Then $a \wedge x<x$. So choose $y \in(a \wedge x, x)$ and $a^{\prime} \in A$ with $y<a^{\prime}$. Then $y \in A \cap[e, x]$ because $a \wedge x=a \wedge a^{\prime}$ and $A$ is chainable. Thus $A \cap[e, x] \neq \varnothing$. A similar argument shows that $x=\sup A \cap[e, m]$ for each $m \geqslant x$. Conversely, if the condition holds, then it is easily shown that $x$ is a quasi supremum of $A$.

A partial order $\leqslant$ on a topological space $(Y, T)$ is said to be semicontinuous if and only if $L(y)$ and $M^{\prime}(y)$ are $T$ closed for each $y \in Y$. Further, $\leqslant$ is monotone if and only if $L(y)$ is connected for each $y \in Y$. The interval topology $I$ for $Y$ has the family of all sets $L(y)$ and $M(y)$ for $y \in Y$ as a subbasis for the closed sets. Evidently $I$ is the smallest topology for which $\leqslant$ is semicontinuous. If $(Y, \leqslant)$ is totally ordered, then the interval topology is usually called the order topology. It is not difficult to prove that a semitree $(X, I)$ is always compact. The following two lemmas were proved in [15].

Lemma 4.2. If $A \subset X$ is chainable and nonempty, then $A$ is closed if and only if $A$ is closed above and below. 
L EMMA 4.3. The following hold:

(i) Both $\varnothing$ and $X$ are closed;

(ii) the intersection of closed sets is closed;

(iii) the union of a finite number of closed sets is closed;

(iv) the semitree order $\leqslant$ is semicontinuous;

(v) each $P \in P$ is closed;

(vi) for each $x \in X$ the set $X \backslash(M(x) \backslash\{x\})$ is closed.

The collection $\{U \subset X \mid X \backslash U$ is closed $\}$ is therefore a topology for $X$ which is called the chain topology. Using Lemma 4.3 it is easy to prove that the chain topology is Hausdorff.

Another way to obtain the chain topology is now developed. Give each chain $P$ in $P$ the natural order topology. The required orders are obtained as follows. If $P$ is an ordered chain, then use $\leqslant_{e}$ restricted to $P$. If $P=[x, y]$ is an unordered chain, then keep the semitree order on $[x \wedge y, y]$ and reverse the semitree order on $[x \wedge y, x]$. Now $P$ is totally ordered in either event, and the corresponding order topology is clearly independent of an interchange of $x$ and $y$ in the order reversal.

An arc is a Hausdorff continuum with exactly two noncutpoints which are called the endpoints of the arc, and an arc need not be separable. A topological space is arcwise connected if and only if any two distinct points are the endpoints of some arc in the space. In the next lemma it is convenient to consider $[x, x]$ as an arc with both endpoints being $x$.

LEMMA 4.4. Each chain is an arc in its natural order topology.

Proof. Let $P \in P$. Then with the order defined above, $P$ is totally ordered and order bounded, complete, and dense. Thus $P$ is a Hausdorf continuum. Now Lemma 1 of Ward [27] implies that $P$ is an arc.

It is easy to see that every pair $P, Q \in P$ satisfies

(a) The order topologies of $P$ and $Q$ agree on $P \cap Q$ and

(b) each $P \cap Q$ is closed in $P$ and $Q$. The strong topology in $X$ induced by $P$ is

$$
T(P)=\{U \subset X \mid U \cap P \text { is open in } P \text { for each } P \in P\} .
$$

Each $P \in P$, as a subspace of $(X, T(P))$, retains its original topology and is a closed subset of the space $X$. Further, $T(P)$ is the largest topology for $X$ that preserves the given topology of each $P \in P$. Also $C \subset X$ is $T(P)$ closed if and only if $C \cap P$ is closed in $P$ for each $P \in P$ [7].

THEOREM 4.5. The chain topology is precisely $T(P)$. 
Proof. Let $C \subset X$ be $T(P)$ closed and let $D=C \cap[x, y] \neq \varnothing$ where $x \leqslant y$. Now $D$ is closed in $[x, y]$, and so it suffices to prove that inf $D$ and sup $D$ are in the closure of $D$ in $[x, y]$. But this follows from the definitions of $\inf D, \sup D$, and the order topology on $[x, y]$. Conversely, suppose that $C$ is closed in the chain topology, and let $[x, y] \in P$. Let $z$ be an element of the closure of $C \cap[x, y]$ in $[x, y]$. Suppose that $z \in(x \wedge y, x]$. Then either $z=\inf C \cap[z, x] \in C$ or $z=\sup C \cap[x \wedge y, z] \in C$. For if neither equality holds, then there is a neighborhood of $z$ in $[x, y]$ that does not meet $C$. This is a contradiction. Similar arguments handle the cases $z=x \wedge y$ and $z \in(x \wedge y, y]$. So $z \in C$, and $C$ is $T(P)$ closed.

So the chain topology is the strong topology induced by the chains. Now $T(P)$ is clearly independent of the semitree order $\leqslant$ used in the definition of closed, and thus the chain topology is independent of the basepoint.

LEMMA 4.6. The chain topology is the strong topology induced by any of the following families:

(i) $\{L(m) \mid m$ is a maximal element in $X\}$;

(ii) $\{L(x) \mid x \in X\}$;

(iii) $\{[x, y] \mid x, y \in X$ and, $x \leqslant y\}$;

(iv) $\mathrm{P}=\{[x, y] \mid x, y \in X\}$;

(v) $\{K \subset X \mid K$ is $T(P)$ compact $\}$.

Proof. Each family is contained in the one below it, and taking strong topologies reverses the inclusions. The first four types of sets induce the order topology on any chain that they happen to contain. Using these facts it is seen that the first four families induce the same strong topology. That the fifth does also is immediate since each $K \subset X$ in (v) is given the topology induced by $T(P)$.

COROLlary 4.7. The space $(X, T(P))$ is a Hausdorff $k$ space.

A topology $T$ for $X$ is weakly order compatible if and only if the following hold:

(a) Each chain in $P$ is $T$ closed;

(b) $T$ induces the order topology on each chain in $P$.

A semitree with a weakly order compatible topology is arcwise connected and Fréchet, and $\leqslant_{e}$ is monotone for any $e$. A fundamental property of such topologies is the following.

LEMma 4.8. Let $T$ be a weakly order compatible topology for $X$. If $z \in$ $U \in T$ and $x<z<y$, then there exist $a, b \in X$ such that $x<a<z<b<y$ and $(a, b) \subset U$. Similar results hold for half open chains. 
PRoof. Now $z \in U \cap[x, y]$ which is open in the order topology on $[x, y]$. So there exist $a, b \in X$ such that $x<a<z<b<y$ and $(a, b) \subset U \cap[x, y] \subset U$. The results for half open chains are proved similarly.

A topology $T$ for $X$ is order compatible at $e$ if and only if the following hold:

(a) $T$ is a weakly order compatible;

(b) for each $x \in X, M(x)$ is $T$ closed.

The term order compatible is due to Wolk [32]. Observe that a topology may be order compatible at one basepoint but not another.

LEMma 4.9. Let $T$ be a topology for $X$. The following are equivalent:

(i) $T$ is order compatible at $e$;

(ii) $I \subset T \subset T(P)$;

(iii) $\leqslant_{e}$ is semicontinuous and for each $x \in X, T$ restricted to $L(x)$ is the order topology on $L(x)$.

PROOF. Observe first that $L(x)$ and $M(x)$ are $T$ closed for each $x \in X$ iff $I \subset T$ iff $\leqslant_{e}$ is semicontinuous. So only the induced topologies need be checked in each implication. Suppose that (i) holds. Then $T \subset T(P)$ since $T$ induces the order topology on the chains. Thus (i) implies (ii). Suppose that (ii) holds. Then $T$ induces the order topology on each $L(x)$ for $x \in X$ since $I$ and $T(P)$ have this property. Thus (ii) implies (iii). Suppose that (iii) holds. But the chain $[x, y]$ is contained in $L(x) \cup L(y)$, and so $T$ induces the order topology on each chain in $P$. So (iii) implies (i), completing the proof.

Let $(Y, \leqslant)$ be a partially ordered set. A subset $S$ of $Y$ is down directed (up directed) if and only if for all $x, y \in S$ there exists $z \in S$ with $z \leqslant x, y$ $(x, y \leqslant z)$. Following McShane and Wolk [32], a subset $C$ of $Y$ is Dedekind closed if and only if whenever $S$ is a down directed subset of $C$ and $y=\inf S$ or $S$ is an up directed subset of $C$ and $y=\sup S$, it follows that $y \in C$. Also $Y$ is Dedekind complete if and only if every down directed subset of $Y$ has an infimum and every up directed subset has a supremum. In a semitree up directed sets are totally ordered, and so any semitree is Dedekind complete. Following Frink [8], a net $\left\{y_{\alpha}\right\}$ in $Y$ is said to converge to $y \in Y$ in the Moore Smith order topology, written $y_{\alpha} \rightarrow y$, if and only if there exist nets $\left\{x_{\alpha}\right\}$ and $\left\{z_{\alpha}\right\}$ such that (a) For each $\alpha, x_{\alpha} \leqslant y_{\alpha} \leqslant$ $z_{\alpha}$; (b) $\sup x_{\alpha}=y=\inf z_{\alpha}$; and (c) if $\alpha \leqslant \beta$, then $x_{\alpha} \leqslant x_{\beta}$ and $z_{\beta} \leqslant z_{\alpha}$. A subset $C$ of $Y$ is closed in the Moore Smith order topology if and only if whenever $\left\{y_{\alpha}\right\} \subset C$ and $y_{\alpha} \rightarrow y$ it follows that $y \in C$. In a semitree the infima and suprema involved in this definition always exist. Notice that Moore Smith closure [8] and convergence in a semitree are not the same as closure and convergence with respect to $T(P)$, but a net that converges with respect to $T(P)$ necessarily possesses a diverse or monotone subnet [9]. 
Let $Y$ be an arcwise connected Hausdorff space. An arc component of $Y$ is any subset of $Y$ which is maximal with respect to being arcwise connected. The space $Y$ is locally arcwise connected if and only if the arc components of open sets are open. Young's arc topology ([28], [34]) is that topology which results from taking the arc components of open sets of the given topology as a basis for the arc topology.

It will now be shown that in a semitree the three concepts of closed just now defined coincide with the concept of closed defined previously.

LEMMA 4.10. The space $(X, T(P))$ is locally arcwise connected.

Proof. Let $A$ be an arc component of a $T(P)$ open set $U$. It suffices to prove that $A \cap[x, y]$ is open in $[x, y]$ where $x \leqslant y$. Let $z \in A \cap(x, y)$. By Lemma 4.8 there exist $a, b \in X$ such that $x<a<z<b<y$ and $(a, b) \subset U$. But $(a, b)$ is arcwise connected and meets $A$. Thus $z \in(a, b) \subset A \cap[x, y]$. The cases $z=x$ and $z=y$ are handled similarly. So $A \cap[x, y]$ is open in the order topology on $[x, y]$, and $A$ is therefore $T(P)$ open.

As a consequence of Lemma 4.10, the chain topology $T(P)$ is precisely Young's arc topology in the space $(X, T(P))$.

THEOREM 4.11. Let $C \subset X$. The following are equivalent:

(i) The set $C$ is $T(P)$ closed;

(ii) the set $C$ is closed in the Moore Smith order topology;

(iii) the set $C$ is Dedekind closed.

Proof. Let $C$ be $T(P)$ closed. Suppose that $\left\{y_{\alpha}\right\} \subset C$ and $y_{\alpha} \rightarrow y$. Let $\left\{x_{\alpha}\right\}$ and $\left\{z_{\alpha}\right\}$ be the nets given by the definition of Moore Smith convergence. Pick some $\alpha_{0}$ and consider $D=C \cap\left[x_{\alpha_{0}}, z_{\alpha_{0}}\right]$. Using the properties of the nets $\left\{x_{\alpha}\right\}$ and $\left\{z_{\alpha}\right\}$, it is easy to see that $y$ is an element of the closure of $D$ in $\left[x_{\alpha_{0}}, z_{\alpha_{0}}\right]$. But $D$ is closed in $\left[x_{\alpha_{0}}, z_{\alpha_{0}}\right]$, and so $y \in D$. Thus $C$ is closed in the Moore Smith order topology, and (i) implies (ii). Suppose now that $C$ is closed in the Moore Smith order topology. Let $S$ be a down directed subset of $C$ and $y=\inf S$. By reversing the order, $S$ can be construed as a net $\left\{y_{\alpha}\right\}$ that converges to $y$ in the Moore Smith order topology. Thus $y \in C$. If $S$ is an up directed subset of $C$ and $y=\sup S$, then analogous reasoning shows that $y \in C$. So $C$ is Dedekind closed, and (ii) implies (iii). Assume now that $C$ is Dedekind closed. If $S$ is a nonempty totally ordered subset of $C$, then $S$ is both down and up directed. So inf $S$, sup $S \in C$, and $C$ is $T(P)$ closed. Therefore (iii) implies (i), completing the proof.

A subset $A$ of $X$ is totally unordered if and only if no two distinct elements of $A$ are comparable under the semitree ordering $\leqslant_{e}$. An important basis for $T(P)$ is now determined. 
Lemma 4.12. A local basis for $T(P)$ at $e$ consists of sets of the form $\bigcup\left\{\left[e, e_{m}\right) \mid m\right.$ is a maximal element of $\left.X\right\}$

where $\left\{e_{m}\right\}$ is totally unordered and $e_{m} \in(e, m]$ for each maximal element $m \in X$.

Proof. Suppose that $e \in U \in T(P)$ and let $C=X \backslash U$. For each $m$ maximal, let $A_{m}=C \cap[e, m]$ and define $e_{m}=\inf A_{m}$ if $A_{m} \neq \varnothing$ and $e_{m}=m$ otherwise. Suppose that $e_{m}<e_{n}$ for $m \neq n$. But then $e_{m} \in C \cap[e, n]=A_{n}$, a contradiction. So $\left\{e_{m}\right\}$ is totally unordered, and clearly $e_{m} \in(e, m]$. Assume that $x \in\left[e, e_{m}\right) \cap C$. Then $x \in A_{m}$, but $x<e_{m}=\inf A_{m}$. Accordingly, $e \in$ $\bigcup_{m}\left[e, e_{m}\right) \subset U$. Finally, $\bigcup_{m}\left[e, e_{m}\right)$ is $T(P)$ open since for each maximal element $n \in X, \bigcup_{m}\left[e, e_{m}\right) \cap[e, n]=\left[e, e_{n}\right)$ which is open in $[e, n]$.

COROLLARY 4.13. If $U$ is a chainable open set containing $e$, then there is a set $\left\{e_{m}\right\}$ of the above type such that

$$
\bigcup_{m}\left[e, e_{m}\right) \subset U \subset \bigcup_{m}\left[e, e_{m}\right] .
$$

Proof. If $y \in U$ and $y \leqslant m$ for $m$ maximal, then $e_{m} \nless y$ since $U$ is chainable, contains $e$ and $y$, and does not contain $e_{m}$.

COROLlary 4.14. A local basis for $T(P)$ at $x \in X$ consists of sets of the form

$$
\bigcup\left\{\left[x, x_{m}\right) \mid m \text { is } \leqslant_{x} \text { maximal in } X\right\}
$$

where $\left\{x_{m}\right\}$ is totally unordered with respect to $\leqslant_{x}$ and $x_{m} \in(x, m]$ for each $\leqslant_{x}$ maximal element $m \in X$.

Combining the bases for $T(P)$ at $x \in X$ given by Corollary 4.14, a basis of chainable sets for $T(P)$ is obtained.

COROLLARY 4.15. The space $(X, T(P))$ is one dimensional in the sense of Menger.

Proof. The boundary of a basic open set $\bigcup_{m}\left[x, x_{m}\right)$ is $\left\{x_{m}\right\}$ which is totally unordered with respect to $\xi_{x}$ and therefore discrete.

The proof of the next lemma is straightforward, but tedious and therefore omitted.

LEMMA 4.16. (i) If $m$ is $\leqslant_{x}$ maximal and if $m \neq e$, then $m$ is $\leqslant_{e}$ maximal.

(ii) If $\left\{x_{m}\right\}$ is totally unordered with respect to $\leqslant_{x}$ and if $x_{m} \xi_{e} x$ for any $m$, then $\left\{x_{m}\right\}$ is totally unordered with respect to $\leqslant_{e}$.

(iii) If $y \leqslant_{e} x$, then

$$
M_{x}(y)=[e, y] \cup \bigcup\left\{[e, m] \mid m \text { is } \leqslant_{e} \text { maximal and } m \wedge_{e} x \leqslant_{e} y\right\} .
$$


For $A \subset X$, let

$$
L(A)=\bigcup\{L(a) \mid a \in A\} \quad \text { and } \quad M(A)=\bigcup\{M(a) \mid a \in A\} .
$$

If $A$ is a family of subsets of $X$, then $T[A]$ denotes the topology which results from taking all $L(A)$ and $M(A)$ for $A \in A$ as a subbasis for the closed sets. The next lemma together with the preceding one shows that it is possible to express a basic open set at $x \in X$ in terms of $L_{e}$ and $M_{e}$.

LEMmA 4.17. Let $U=\bigcup_{m}\left[x, x_{m}\right)$ be a basic open set at $x$ as given in Corollary 4.14. Then the following hold:

(i) If there exists $x_{m_{0}} \leqslant_{e} x$, then

$$
U=X \backslash\left[M_{e}\left(\left\{x_{m} \mid x_{m} \sharp_{e} x\right\}\right) \cup M_{x}\left(x_{m_{0}}\right)\right] ;
$$

(ii) otherwise, $U=X \backslash M_{e}\left(\left\{x_{m}\right\}\right)$.

COROLlary 4.18. Let A consist of the subsets of $X$ which are totally unordered with respect to $e$. Then $T(P) \subset T[A]$.

Equality need not hold in Corollary 4.18 since $L_{e}(A)$ is not necessarily $T(P)$ closed for each $A \in A$.

A topology $T$ for $X$ is strongly order compatible at $e$ if and only if $T$ is order compatible at $e$ and $M(x) \backslash\{x\}$ is $T$ open for each $x \in X$. A semitree with a strongly order compatible topology will turn out to be the best candidate in this paper for the title of noncompact tree. By Lemma 4.3, $T(P)$ is strongly order compatible at any basepoint. Also the topologies in question are now Hausdorff (see Lemma 4.19). Recall that a branch $B$ at a point $x \in X$ was defined in $\S 3$ in terms of a particular semitree order $\leqslant_{e}$.

LEMMA 4.19. Let $T$ be a topology for $X$ which is strongly order compatible at $e$ and let $B$ be a branch at $x \in X$. Then

(i) $B$ as well as any union of branches at $x$ is $T$ closed;

(ii) $B \backslash\{x\}$ is $T$ open;

(iii) $(X, T)$ is completely Hausdorff.

Proof. Suppose that $y \notin B$. Then, since $M(x)$ is closed and $B \subset M(x)$, it may be assumed that $y \in M(x) \backslash\{x\}$. Then there is a $z$ such that $x<z<y$. Now $y \in M(z) \backslash\{z\}$ and $(M(z) \backslash\{z\}) \cap B=\varnothing$ by Lemma 3.2(i) and (ii). Thus $B$ is closed, and any union of branches at $x \in X$ is analogously shown to be closed. That $B \backslash\{x\}$ is open follows from Lemma 3.2(v). To show (iii) let $x, y \in X$ with $x \neq y$. If $x<y$, then there exist $a, b \in X$ with $x<a<$ $b<y$. Then $X \backslash M(a)$ and $M(b) \backslash\{b\}$ are the desired open sets. If $x$ and $y$ are not comparable, then there exist $a \in(x \wedge y, x)$ and $b \in(x \wedge y, y)$. Then $M(a) \backslash\{a\}$ and $M(b) \backslash\{b\}$ are the required open sets. 
Observe the strength of Lemma 4.19. Part (i) implies that $M(x)$ is $T$ closed for each $x \in X$ by Corollary 3.3. For each $x \in X, M(x) \backslash\{x\}=$ $\bigcup\{B \backslash\{x\} \mid B$ is a branch at $x\}$. Thus $B \backslash\{x\}$ being $T$ open for each $x \in X$ and each branch $B$ at $x$ in fact implies that $M(x) \backslash\{x\}$ is $T$ open for each $x \in X$. The next theorem shows that the concept of a strongly order compatible topology is independent of the basepoint. Hence all reference to the basepoint can be dropped when dealing with strongly order compatible topologies.

THEOREM 4.20. If $T$ is a topology for $X$ which is strongly order compatible at one basepoint, then $T$ is strongly order compatible at any other basepoint.

Proof. Assume that $T$ is strongly order compatible at $e$ and let $f$, $x \in X$. The notations $\leqslant,<$, and $\wedge$ are taken with respect to $e$ throughout the following argument. If $x=f$, then $M_{f}(x)=X$ and $M_{f}(x) \backslash\{x\}=X \backslash\{x\}$ which are obviously $T$ closed and $T$ open, respectively. If $x \notin f$, then it will be shown that $M_{f}(x)=M_{e}(x)$, and so $M_{f}(x) \backslash\{x\}=M_{e}(x) \backslash\{x\}$. So both sets again have the required properties. To show that $M_{f}(x)=M_{e}(x)$, observe that $y \in M_{f}(x)$ iff $x \in[f, y]$ iff $x \in[f \wedge y, y]$ iff $x \in[e, y]$ iff $x \leqslant y$ iff $y \in M_{e}(x)$ where $x \notin f$ has been used twice. Suppose finally that $x<f$ and let $B$ be the branch at $x$ containing $f$ given by Lemma 3.2(iii). Then it will be shown that $M_{f}(x)=X \backslash(B \backslash\{x\})$, and so $M_{f}(x) \backslash\{x\}=X \backslash B$. Applying Lemma 4.19(ii), both sets again have the necessary properties. To prove the stated equality, note that $y \in M_{f}(x)$ iff $x \in[f, y]$ iff $x \in[f \wedge y, f)$ iff $f \wedge y \leqslant x$ iff $f \wedge y \notin B \backslash\{x\}$ iff $y \notin B \backslash\{x\}$ iff $y \in X \backslash(B \backslash\{x\})$. The fourth iff is equivalent to $x<f \wedge y$ iff $f \wedge y \in B \backslash\{x\}$ which follows from Lemma 3.2(i).

A connected space $Y$ is hereditarily unicoherent if and only if whenever $A$ and $B$ are closed connected subsets of $Y$, it follows that $A \cap B$ is connected. Now let $Y$ be an arcwise connected Hausdorff space. Then $Y$ is acyclic if and only if any two distinct points are the endpoints of exactly one arc in the space. Further, $Y$ is nested if and only if the union of each nest of arcs in $Y$ is contained in an arc in $Y$. An acyclic continuum need not be nested, and a nested continuum need not be hereditarily unicoherent. Also $Y$ is dendritic if and only if $Y$ is locally connected and any two distinct points can be separated in $Y$ by the omission of some third point. A dendritic space need not be nested, and a locally connected nested continuum need not be dendritic (see §5(c)).

THEOREM 4.21. Let $T$ be a strongly order compatible topology for $X$. Then the following hold:

(i) Every arc in $X$ is a chain;

(ii) arcwise connectivity, chainability, and $T$ connectivity are all equivalent for subsets of $X$; 
(iii) the intersection of connected sets is connected;

(iv) the space $(X, T)$ is acyclic, nested, and in fact hereditarily unicoherent.

Proof. Let $A$ be an arc in $X$ with endpoints $x$ and $y$. Suppose that $z \in[x, y] \backslash A$. Without loss of generality, $z \neq x \wedge y$ since $[x, y] \backslash A$ is open in the connected set $[x, y]$. So it can be assumed that $z \in(x \wedge y, x)$, for example. But $(M(z) \backslash\{z\}) \cap A=M(z) \cap A$ is then a clopen, nonempty, proper subset of the connected set $A$. This is a contradiction. So $[x, y] \subset A$ Suppose now that $a \in A \backslash[x, y]$. But $A$ is an arc and therefore has the order topology induced by some total order $\leqslant^{*}$. Now work inside $A$ letting $M_{A}(a)=$ $\left\{z \in A \mid a \leqslant^{*} z\right\}$. But $\left(M_{A}(a) \backslash\{a\}\right) \cap[x, y]=M_{A}(a) \cap[x, y]$ is then a clopen, nonempty, proper subset of the connected set $[x, y]$. This is a contradiction. So $A=[x, y]$ is a chain, and this finishes the proof of (i). Now (i) implies that arcwise connectivity and chainability are equivalent, and obviously arcwise connectivity implies $T$ connectivity. Now let $A$ be $T$ connected and let $x, y \in A$. Suppose that $z \in[x, y] \backslash A$. Let $x \wedge y=i$ and suppose first that $z \neq i$. Then

$$
A=[(X \backslash M(z)) \cap A] \cup[(M(z) \backslash\{z\}) \cap A]
$$

would be a separation of the connected set $A$. Second, suppose that $z=i$. Then with no loss of generality $A \subset M(i) \backslash\{i\}$, for otherwise use the separation given above. Let $B$ be the branch at $i$ containing $x$ given by Lemma 3.2(iii), and let $C$ be the union of all other branches at $i$. Then $B \backslash\{i\}$ and $C \backslash\{i\}$ are open by Lemma 4.19(ii). Thus

$$
A=[(B \backslash\{i\}) \cap A] \cup[(C \backslash\{i\}) \cap A]
$$

would be a separation of $A$ since $M(i) \backslash\{i\}=(B \cup C) \backslash\{i\}, B \cap C=\{i\}$, and $y \in C \backslash\{i\}$. Therefore, $A$ must be chainable, and this proves (ii). Clearly, (ii) implies (iii) by Lemma 2.1(iv). Finally, Axioms 1 and 5 together with (i) and (iii) imply (iv).

CoROllaRY 4.22. If $T$ is a strongly order compatible topology for $X$, then the arc components of the $T$ open sets $M(x) \backslash\{x\}$ are $T$ open for each $x \in X$.

Proof. The arc components of the $T$ open sets $M(x) \backslash\{x\}$ where $x \in X$ are exactly the $B \backslash\{x\}$ used in the proof of Theorem 4.21 where $B$ is some branch at $x$.

In spite of Lemma 4.10 and Corollaries 4.14 and 4.22, a semitree $X$ with a strongly order compatible topology need not be locally arcwise connected, although there is a converse. A chain component of a set $A \subset X$ is any subset of $X$ which is maximal with respect to being chainable. The space $(X, T)$ is locally chainable if and only if the chain components of $T$ open sets are $T$ open 
Either Corollary 4.14 or Lemma 4.10 and Theorem 4.21 imply that $(X, T(P))$ is locally chainable.

THEOREM 4.23. If $(X, T)$ is Fréchet and locally chainable, then $L(x)$, $M(x)$, and $X \backslash(M(x) \backslash\{x\})$ are $T$ closed for each $x \in X$.

Proof. If $x \in X$ and $B$ is a branch at $x$, then $B \backslash\{x\}$ is a chain component of $X \backslash\{x\}$ and is therefore open. Thus $M(x) \backslash\{x\}$ is open. Also, $X \backslash M(x)$ is a chain component of $X \backslash\{x\}$ and is therefore open for each $x \in X$. Finally,

$X \backslash L(x)=(M(x) \backslash\{x\})$

$\cup \cup\{B \backslash\{y\} \mid B$ is a branch at $y \in[e, x)$ and $B \cap[e, x]=\{y\}\}$ by Corollary 3.3 , and so $L(x)$ is $T$ closed.

Branches at $x \in X$ could in fact be defined in terms of chain components of $M(x) \backslash\{x\}$. Theorem 4.21 permits the following strengthening of a fixed point theorem that appeared in [15].

THEOREM 4.24. Let $(X, T)$ be a nested space where $T$ is a strongly order compatible topology. If $F: X \rightarrow X$ is lower semicontinuous and point connected, then $F$ has a fixed point.

A partial order $\leqslant$ on a topological space $(Y, T)$ is said to be continuous if and only if $\leqslant$ is a closed subset of $Y \times Y$.

Lemma 4.25. Let $T$ be a strongly order compatible topology for $X$. Then the following hold:

(i) Any two distinct points in $X$ can be separated by the omission of a third point;

(ii) the semitree order $\leqslant$ is continuous.

Proof. Let $x, y \in X$ where $x \neq y$. If $x$ and $y$ are comparable, then any $z \in(x, y)$ separates $x$ and $y$ by its removal. If $x$ and $y$ are not comparable, then $x \wedge y$ separates $x$ and $y$ by its removal. To see the latter statement, use $X \backslash M(x \wedge y)$ and the branches at $x \wedge y$. The proof of (ii) is standarả [25].

Observe that continuity of a semitree order need not imply strong order compatibility of the topology [25]. Moreover, for a given weakly order compatible topology, the semitree order $\leqslant_{e}$ need not in general be continuous or even semicontinuous for any $e \in X[20]$.

But if $\leqslant_{e}$ is semicontinuous with respect to a weakly order compatible topology $T$ for any $e \in X$, then $T$ is strongly order compatible [20].

COROLLARY 4.26. If $(X, T)$ is locally chainable and $T$ is a weakly order 
compatible topology, then $(X, T)$ is dendritic, and hence every continuum contained in $X$ is a tree [26].

Let $T_{0}$ denote the topology for $X$ with subbasis consisting of all sets of the form $X \backslash L(x), X \backslash M(x)$, or $M(x) \backslash\{x\}$ where $x \in X$.

TheOREM 4.27. The space $\left(X, T_{0}\right)$ is compact Hausdorff. Hence, $\left(X, T_{0}\right)$ is a tree and is therefore locally chainable.

Proof. Observe first that $T_{0}$ is a strongly order compatible topology because $I \subset T_{0} \subset T(P)$. Second, by Alexander's subbasis lemma it suffices to prove that each nonvoid family $F$ of subbasic closed sets with fip has nonempty intersection. Third, if $F$ contains some $L(x)$, then clearly $\bigcap F \neq \varnothing$ since $L(x)$ is $T_{0}$ compact. So assume that $F$ contains no $L(x)$ for ' $x \in X$. Since $F$ has fip, the set $S=\{x \mid M(x) \in F\}$ is totally ordered. Also $S$ may be assumed to be nonempty since otherwise $e \in \bigcap F$. Let $s=\sup S$, and then $s \in \bigcap\{M(x) \mid M(x) \in F\}$. It is then easy to see that $s \in \bigcap F$. For suppose that $s \in M(x) \backslash\{x\}$ where $X \backslash(M(x) \backslash\{x\}) \in F$. Since $s=\sup S$ there exists $y \in S \cap(x, s)$. But then $M(y) \cap(X \backslash(M(x) \backslash\{x\}))=\varnothing$, a contradiction. Thus $\left(X, T_{0}\right)$ is compact Hausdorff. Hence, $\left(X, T_{0}\right)$ is a tree [24] and is therefore locally connected ([24], [25]).

THEOREM 4.28. If $C \subset X$ is chainable, then $C$ is $T_{0}$ closed if and only if $C$ is $T(P)$ closed.

Now $T_{0}$ is clearly the minimal strongly order compatible topology. Thus there is exactly one compact strongly order compatible topology, and so there is exactly one way to make a semitree into a tree that is compatible with the chain structure. Therefore $T_{0}$ will be called the tree topology for $X$. Note that $\left(X, T_{0}\right)$ has finite boundaries [24].

The families of weakly order compatible topologies studied in this section form a spectrum (inclusion chain) of topologies that are compatible with the chain structure of the semitree. They include the order compatible topologies (equivalently, $\leqslant$ is semicontinuous), the topologies for which $\leqslant$ is continuous, the strongly order compatible topologies, and the topologies for which the semitree is locally chainable. Of particular interest are $I$, the minimal order compatible topology; $T_{0}$, the minimal strongly order compatible topology; and $T(P)$, the maximal strongly order compatible topology (also the maximal weakly order compatible topology). The minimal weakly order compatible topology has $P$ as a subbasis for the closed sets. A semitree with a topology from this spectrum will be called a topological semitree.

5. Examples. Many interesting examples of topological semitrees are presented in this section, and the spectrum of topologies described in the last section 
is examined in a few specific cases. Some other examples are given for the sake of contrast.

(a) Arcs. The unit interial $I$ is the simplest semitree, and being the only metric arc, it is usually called the real arc. More generally, any arc admits the structure of a semitree wherein the chains are the subcontinua and $e$ is an endpoint. Any nondegenerate subcontinuum of an arc is an arc in the induced topology. The chain, interval, order, and tree topologies all coincide in an arc. So there is exactly one weakly order compatible topology for an arc, and it is locally chainable. All arcs are nested, and they are the basic building blocks for semitrees. The long interval is perhaps the simplest nonmetric arc, and it serves as a counterexample to several conjectures about properties possessed by semitrees [22]. The unit square $I^{2}$, ordered lexicographically and given the order topology, is an arc [22]. More generally, any power of unit intervals, $I^{m}, m$ a cardinal, is an arc when similarly constrained. The arc $I^{\aleph 0}$ is particularly interesting for it enjoys a property common to both the real arc and the pseudo arc. By a theorem of Arens ([1], [23]), $I^{\aleph_{0}}$ is hereditarily equivalent; that is, every nondegenerate subcontinuum of $I^{\aleph_{0}}$ is homeomorphic to $I^{\aleph_{0}}$. It would be interesting to have a theorem that gives constructive procedures for obtaining all arcs.

(b) Nested spaces. Let $(X, T)$ be a nested space and let $P$ be the family of arcs in $X$ where $\{x\}$ is considered to be an arc with both endpoints being $x$. Mohler has proved that nested spaces are acyclic [14]. An interesting question is whether the Hausdorff assumption in the definition of a nested space is necessary for the space to be acyclic. It is easily shown that $(X, P)$ is a semitree and $(X, T)$ induces the order topology on the chains. So $T$ is a weakly order compatible topology, and $T \subset T(P)$. Also $A \subset X$ is an arc if and only if it is a chain. The cutpoint order with basepoint $e$ [30] is contained in the semitree order $\leqslant_{e}$. These results and some converses will be summarized in $\S 7$. The $B$ spaces studied by Holsztyński [11] are a special type of nested space first considered by Borsuk [3]. Nested spaces were introduced by Young [34].

Other special cases of nested spaces include the arboroids of Ward [27]. An arboroid is an arcwise connected hereditarily unicoherent Hausdorff continuum. Among the arboroids are the dendroids (metric arboroids), the trees (locally connected arboroids), and the dendrites (metric trees). The arboroids also include the generalized trees (smooth arboroids [5]) of Ward [25] and those arcwise connected continua that are hereditarily divisible by points [14]. It will now be proved that every arboroid is nested and thus is a semitree. The crucial step is to prove that arboroids are hereditarily decomposable, and this follows by putting together theorems of Bellamy, Harris, and Ward. Bellamy recently proved [2] that any indecomposable continuum can be mapped continuously onto the 
Knaster continuum [30]. Harris showed that the continuous image of an arc is arcwise connected [10]. Ward proved that any subcontinuum of an arboroid is arcwise connected [27]. In particular, no arcwise connected continuum is indecomposable. Now let $X$ be an arboroid and let $P$ consist of the arcs in $X$. Ward has shown that $X$ has a natural order theoretic characterization in terms of an order $\leqslant$ that turns out to be a semitree order [27]. Using this order Ward proved that every nonempty totally ordered subset of an hereditarily decomposable arboroid has a supremum. Since it is now known that every arboroid is hereditarily decomposable, $(X, \leqslant)$ satisfies Lemma 2.4(ii), and from this fact the nested property follows readily.

The following natural characterizations of some acyclic spaces in terms of semitrees follow from results of $\S 4$, Smithson [20], and Ward ([24]-[27]).

TheOREM 5.1. Let $(X, P)$ be a semitree and let $T$ be a weakly order compatible Hausdorff topology for $X$. Then the following hold:

(i) $(X, T)$ is an arboroid if and only if $(X, T)$ is compact and every subcontinuum of $X$ is chainable (if and only if $(X, T)$ is acyclic, compact, and every subcontinuum of $X$ is arcwise connected);

(ii) $(X, T)$ is a generalized tree if and only if $(X, T)$ is an arboroid and $\leqslant_{e}$ is continuous for some $e$;

(iii) $(X, T)$ is dendritic if and only if $(X, T)$ is locally chainable;

(iv) $(X, T)$ is a tree if and only if $(X, T)$ is compact and $\leqslant_{e}$ is semicontinuous for any $e$.

Suppose further that $T$ is strongly order compatible. Then

(v) $(X, T)$ is dendritic if and only if $(X, T)$ is locally connected;

(vi) $(X, T)$ is a tree if and only if $(X, T)$ is compact.

It should be again pointed out that dendritic spaces need not be nested. Parts (iii) and (v) above are only concerned with those dendritic spaces that happen to be nested.

(c) Fans. Let $(X, P)$ be a semitree. The order of a point $x \in X$ is the maximum possible number of chains in $P$ that have $x$ as an endpoint and do not intersect otherwise. This cardinal number will be denoted by Ord (x). A point of order one is an endpoint of the semitree, and a point of order three or greater is a branch point. It is easy to see that every maximal element is an endpoint, and the only other possible endpoint is $e$. Conversely, an endpoint is either $e$ or $\leqslant_{e}$ maximal.

A fan is a semitree in which the infimum of any two noncomparable elements is $e$. Equivalently, a fan is a semitree with at most one branch point. Intuitively, a fan is just a bundle of arcs tied together at $e$. A finite (countable) fan or semitree is one that has only finitely (countably) many endpoints. If a 
fan is given a topology that is weakly order compatible, then that topology is order compatible at $e$. Charatonik's concept of fan $([5],[6])$ is a fan in the present sense.

Closed stars in real vector spaces are fans. Let $V$ be a real vector space and let $e \in V$. A line $L$ through $e$ is a set $L=e+U$ where $U$ is a one dimensional linear subspace of $V$. A closed line segment is a set of the form

$$
\{z \in V \mid z=\lambda x+(1-\lambda) y \text { and } 0 \leqslant \lambda \leqslant 1\} \text { where } x, y \in V .
$$

A subset $S$ of $V$ is a closed star at $e$ if and only if $e \in S$ and each line $L$ through $e$ intersects $S$ in a closed line segment. The arcs in a closed star in a topological vector space are real arcs, but the closed star itself need not be topologically closed or compact.

Let $B$ denote the closed infinite broom [22] which is just a bundle of $\aleph_{0}$ real arcs embedded in the plane (including a limit line) and radiating from the origin. Let $D$ denote the unit disc in the plane at the origin. Now $D$ can be thought of as a bundle of $c$ real arcs emanating from the origin in the plane, where $c$ is the cardinality of the reals. Consequently, $B$ and $D$ have a similar semitree structure, and the resulting spectra of order compatible topologies are very similar. The similarities as well as some subtle differences will be pointed out. For the moment let $X$ stand for either $B$ or $D$. Let $e$ be the origin in $X$. The ordered chains in $X$ are the radial line segments that contain $e$ when extended. Keeping in mind that $X$ is to be a fan and in fact a closed star at $e$ in the plane, the other chains are defined in the obvious way. The Euclidean subspace topology $T_{E}$ is a compact Hausdorff order compatible topology that is not strongly order compatible. The interval topology $I$ is not Hausdorff, and the chain topology $T(P)$ is neither compact nor metrizable. Ward made $D$ into a dendritic space $\left(D, T_{W}\right)$ which admits no compactification as a tree [26], and the same construction applies to $B$. In fact Ward's proof shows that $(X, T)$ admits no compactification as a tree when $T$ is any order compatible topology such that $(X, T)$ is not a tree to begin with. Young's arc topology in the space $\left(B, T_{E}\right)$ is exactly $T_{W}$, and the same is true of $\left(D, T_{E}\right)$ if the chain components of $T_{E}$ open sets are taken as a basis for the arc topology. Now

$$
I \subset T_{E} \subset T_{W} \subset T(P)
$$

where each inclusion is strict. Infinitely many order compatible topologies between $T_{E}$ and $T(P)$ can be obtained by changing the topology on $D$ in sectors of angle $\theta(0<\theta<2 \pi)$ from $T_{E}$ to $T(P)$, and none of these are related to $T_{W}$ under inclusion. Now $X$ can also be thought of as the 
axes in the product $I^{m}\left(m=\aleph_{0}, c\right)$. Then the subspace topology induced by the product topology on $I^{m}$ is exactly the tree topology $T_{0}$, and

$$
I \subset T_{0} \subset T_{W} \subset T(P)
$$

where all inclusions are strict. There is one final topology on $D$ of interest; namely, the subspace topology $T_{C}$ induced by the topology on $I^{c}$ that restricts at most countably many coordinates. Then

$$
I \subset T_{0} \subset T_{C} \subset T(P)
$$

where each inclusion is strict. No other inclusions hold among any of the topologies discussed above except those indicated. Observe that $\left(B, T_{0}\right)$ is a dendrite, whereas $\left(D, T_{0}\right)$ is a nonmetric tree with real arcs. The two cell has been made into a semitree in two essentially different ways: as an arc $I^{2}$ whose topology is not related to the Euclidean topology, and as a bundle $D$ of $c$ real arcs wherein the Euclidean topology is order compatible.

Certain types of cones provide further interesting examples of fans. See [7] for the definition of a cone. The Cantor fan is just the cone over a Cantor set. The cone over a pseudo arc is a nested continuum that is no: hereditarily unicoherent ([10], [34]). Let $M$ denote Mardešićs locally connected continuum which contains no proper locally connected subcontinuum [12]. The cone over $M$ is a locally connected nested continuum that is not dendritic. Similar examples are obtained by taking cones over other continua that contain no arcs; for example, the product of $n$ pseudo arcs, $n$ a positive integer [35]. It is interesting to ask whether an acyclic locally connected continuum must be nested [26].

The spectrum of order compatible topologies also collapses for a finite fan or semitree. Let $X$ denote a finite semitree. If $T$ is a weakly order compatible topology for $X$, then $T$ is order compatible at any $e \in X$ by Lemma 4.16(iii). Now $X$ contains no infinite totally unordered set, and so $X$ possesses a unique weakly order compatible topology [18] that must be locally chainable. This topology is $T_{0}$, and $\left(X, T_{0}\right)$ is a tree. If $X$ has real arcs, then it is easy to see either directly or by using a lemma of Wolk [33] that $\left(X, T_{0}\right)$ satisfies the second axiom of countability. So $\left(X, T_{0}\right)$ is metrizable [33] and thus is a dendrite. Note that an arbitrary semitree can have infinitely many endpoints and still have a compact strongly order compatible topology.

(d) Combs. The combs form another important family of semitrees and include the fans [5]. Many combs have a common semitree substructure that is constructed as follows. For $n=1,2, \cdots$, erect at $(1 / n, 0)$ in the plane a perpendicular interval of length one. Let $C$ denote the union of the 
erected intervals and the unit interval, and let $T_{E}$ denote the Euclidean subspace topology for $C$. Then $\left(C, T_{E}\right)$ is called an infinite comb. A closed infinite comb is obtained by attaching a limit line to $C$. Let $e$ denote the origin $(0,0)$ and let $f$ denote the point $(1,0)$. Now $C$ is made into a semitree by letting $P$ consist of the arcs in $C$, but $C$ also has the structure of a one dimensional simplicial complex $K$. The Whitehead topology is the strong topology $T(K)$ induced by $K$ where each simplex in $K$ is given the order topology. Define another topology $T_{S}$ for $C$ as follows. Take the usual open sets in $C \backslash\{e\}$. A basic open set containing $e$ will consist of the points in $C$ that are to the left of a line $x=c$ and either above a line $y=b$ or below a line $y=a$ where $a, b, c \in(0,1)$ and $a<b$. The basis at $e$ is obtained by letting $a, b$, and $c$ vary. Then $T_{S}$ is a strongly order compatible topology, but $\left(C, T_{S}\right)$ is not locally connected. Also

$$
I_{e} \subset I_{f}=T_{0} \subset T_{S} \subset T_{E} \subset T(P) \subset T(K)
$$

where each inclusion is strict and $I_{e}\left(I_{f}\right)$ denotes the interval topology determined by $\leqslant_{e}\left(\leqslant_{f}\right)$. These topologies admit particularly nice subbases for the closed sets. For example, $I_{e}=T\left[A_{1}\right], T_{0}=T\left[A_{0}\right], T(P)=$ $T\left[A_{e}\right]$, and $T(K)=T\left[A_{f}\right]$ where the notation of $\S 4$ is used and where $A_{1}$ consists of the singletons in $C, A_{0}$ consists of $A_{1}$ and the $T_{0}$ closures of sets of $\leqslant_{e}$ maximal elements in $C$, and $A_{e}\left(A_{f}\right)$ consists of the subsets of $C$ that are totally unordered with respect to $\leqslant_{e}\left(\leqslant_{f}\right)$. The first equality is trivial, and the second equality is a consequence of the main theorem in [21]. The third equality follows from Corollary 4.18 and the fact that $L_{e}(A)$ is $T(P)$ closed for each $A \in A_{e}$. The fourth equality follows since $\{e\}$ is an open set in both topologies.

The Hilbert cube $I^{\kappa_{0}}$ (or any cell for that matter) can be given a comblike semitree structure distinct from the arc and closed star structures mentioned above. For $a, b \in I^{\aleph_{0}}$ define $a \leqslant b$ if and only if either $a_{i}=0$ for all $i \in \aleph_{0}$ or else there exists $n \in \aleph_{0}$ such that

(a) $a_{n} \leqslant b_{n}$;

(b) for all $i>n, a_{i}=b_{i}$;

(c) if $n>0$, then $a_{0}=a_{1}=\cdots=a_{n-1}=0$.

Then $\left(I^{\aleph_{0}}, \leqslant\right)$ is a partially ordered set meeting the requirements of Theorem 2.7.

(e) Other examples. Using Axioms 1-6 of $\S 2$ as instructions and the examples in (a)-(d) above as construction materials, the methods of identification yield other examples. In fact, it will be shown in $\$ 7$ that every semitree is an identification space of an appropriate fan when the topologies are chosen naturally. Examples obtained by identification can be found in nearly 
all of the references listed at the end of the paper.

Perhaps the simplest nonnested acyclic continuum is the topologist's $\sin 1 / x$ circle [28]. Other examples can also be found in the references. The following result leads to even more examples.

Lemma 5.2. Let $X$ be a semitree, let $T$ be a strongly order compatible topology for $X$, and let $m \in X \backslash\{e\}$. The following are equivalent:

(i) $m$ is $\leqslant_{e}$ maximal;

(ii) $m$ is an endpoint (that is, $\operatorname{Ord}(m)=1$ );

(iii) $m$ is a noncutpoint.

It is necessary to assume that $T$ is strongly order compatible in Lemma 5.2 since the Cantor swastika is a dendroid without cutpoints [13]. Also the order of $m \in X$ in Lemma 5.2 may be taken in the classical sense [4] since every arc in $(X, T)$ is a chain by Theorem 4.21. Finally, (ii) and (iii) in Lemma 5.2 are equivalent for all $m \in X$.

COROLlARY 5.3. If $(X, T)$ is a nondegenerate space without cutpoints (noncutpoints), then it is impossible to give $X$ the structure of a semitree wherein $T$ is a strongly (weakly) order compatible topology.

The complement of any set of maximal elements in a semitree is chainable, whereas the removal of any pair of distinct points disconnects the unit circle. Thus it is impossible to give the unit circle a semitree structure and have the Euclidean topology be weakly order compatible. It is conjectured that this is true of any $n$ sphere, $n$ a positive integer. Using the above ideas, it is easily seen that every nondegenerate nested space has at least two noncutpoints.

6. Characterizations of chains. In this section the chains in a semitree are characterized leading to a description of arcs. Let $(X, P, \leqslant)$ be an algebraic semitree throughout this section.

Lemma 6.1. Suppose that $\varnothing \neq A \subset X$. Then the following hold:

(i) If $A$ is closed above, then the quasi suprema of $A$ are exactly the maximal elements of $A$;

(ii) if $A$ is closed below, then $\inf A$ is the least element of $A$;

(iii) if $a_{0} \in A$, then $A$ has a quasi supremum $s$ such that $a_{0} \leqslant s$;

(iv) if $\sup A$ exists, then $\sup A$ is a quasi supremum of $A$.

Proof. The proof of (i) is not difficult, and part (ii) is trivial. To prove part (iii) let $a_{0} \in A$. By Wallace's principle let $M$ be a maximal $\leqslant$ totally ordered subset of $A$ that contains $a_{0}$. Now let $s=\sup M$. Then $s$ is a quasi supremum of $A$ and $a_{0} \leqslant s$. To prove (iv) suppose that $s=\sup A$ 
exists. Then for all $a \in A, s \varangle a$ since $s$ is in fact an upper bound of $A$. Assume that $y \in X$ satisfies $y \varangle a$ for all $a \in A$. If $y<s$, then $A \subset L(s)=L(y) \cup[y, s]$ and $A \cap(y, s]=\varnothing$ imply that $A \subset L(y)$, a contradiction. Thus $s$ is a quasi supremum of $A$.

Unfortunately, not all of the quasi suprema of a set $A \subset X$ can be obtained in the manner of the proof of Lemma 6.1(iii) unless $A$ is chainable.

Lemma 6.2. Suppose that $\varnothing \neq A \subset X$. Then the following are equivalent:

(i) $A$ has exactly one quasi supremum;

(ii) $A$ is totally ordered;

(iii) $\sup A$ exists.

Proof. For (i) implies (ii), use Lemma 6.1(iii). For (ii) implies (iii), use Lemma 2.4(ii). For (iii) implies (i), suppose that $\sup A=s$ exisis. Then $s$ is a quasi supremum of $A$ by Lemma 6.1(iv). Assume that $t$ is a quasi supremum of $A$. If $t \& s$, then there exist $x \in(s \wedge t ; t)$ and $a \in A$ with $x<a$. But $x<a \leqslant s$, a contradiction. So $t \leqslant s$, and then $t=s$ follows similarly. Thus $s$ is the only quasi supremum of $A$.

COROLlary 6.3. If $A$ is closed above and totally ordered, then $\sup A$ is the greatest element of $A$.

Lemma 6.4. Suppose that $\varnothing \neq P \subset X$. Then $P$ is an ordered chain if and only if $\boldsymbol{P}$ is chainable, closed, and totally ordered.

PRoof. That an ordered chain has the stated properties follows from Lemmas 2.3 and 4.3. Suppose that $P \subset X$ is chainable, closed, nonempty, and totally ordered. Let $i=\inf P$ and $s=\sup P$. Then $[i, s] \subset P$ since $P$ is chainable and closed. If $p \in P$, then $i \leqslant p \leqslant s$ and so $p \in[i, s]$. Thus $P=[i, s]$ is an ordered chain.

The conditions in Lemma 6.4 and the next lemma may be replaced by the equivalent conditions in Lemmas 4.2 and 6.2.

Lemma 6.5. Suppose that $\varnothing \neq P \subset X$. Then $P$ is an unordered chain if and only if $P$ is chainable and closed and $P$ has exactly two quasi suprema $x$ and $y$ where inf $P=x \wedge y$.

Proof. Assume that $P=[x, y]$, for $x, y \in X$, is an unordered chain. Then $P$ is chainable and closed by Lemmas 2.3 and 4.3. Now $x$ and $y$ are quasi suprema of $P$. For clearly $x \not p$ for all $p \in P$ as $P \subset L(x) \cup L(y)$. Suppose that $z<x$. Then $z \vee(x \wedge y)<x$. So choose $q \in(z \vee(x \wedge y), x)$, and then $z<q \in P$. So $x$ is a quasi supremum of $P$, and similarly $y$ is a quasi supremum of $P$. If $z$ is a quasi supremum of $P$, then $z \in P$. So suppose that $z \leqslant x$. Then $z \neq x$ is impossible. Thus $x$ and $y$ 
are the only two quasi suprema of $P$, and $x \wedge y=\inf P$ by Lemma 2.5. Suppose conversely that $P \subset X$ is chainable, closed, and nonempty and that $P$ has exactly two quasi suprema $x$ and $y$ where inf $P=x \wedge y$. Then $x, y \in P$ since $P$ is closed and $[x, y] \subset P$ since $P$ is chainable. Suppose that $p \in P$. By Lemma 6.1(iii), it can be assumed that $p \leqslant x$. Therefore $x \wedge y=\inf P \leqslant$ $p \leqslant x$, and so $p \in[x, y]$. Thus $P=[x, y]$ is an unordered chain.

A triod $T$ in $X$ is a subset $T$ of $X$ that is the union of three nondegenerate chains each having a point $a$ as an endpoint and not intersecting otherwise. The point $a$ is of course a branch point of $X$. A subset $A$ of $X$ is atriodic if and only if $A$ contains no triods. There is the following easy characterization of triods in semitrees.

LemmA 6.6. Let $a, a_{1}, a_{2}, a_{3} \in X$ be all distinct. Then $T=\bigcup_{n=1}^{3}\left[a, a_{n}\right]$ is a triod if and only if the following hold:

(i) There exist $i, j \in\{1,2,3\}$ such that $a=a_{i} \wedge a_{j}$;

(ii) if $k \in\{1,2,3\} \backslash\{i, j\}$ and $a \leqslant a_{k}$, then $a_{i} \wedge a_{k}=a=a_{j} \wedge a_{k}$.

THEOREM 6.7. Suppose that $\varnothing \neq P \subset X$. Then $P$ is a chain if and only if $P$ is atriodic, chainable, and closed.

Proof. Assume that $P=[\dot{x}, y]$ is a chain where $x, y \in X$. Now $P$ is known to be chainable and closed. Suppose that $T=\bigcup_{n=1}^{3}\left[a, a_{n}\right]$ is a triod in $P$. Let $i, j, k$ be as in Lemma 6.6. Then $a_{i}$ and $a_{j}$ are not comparable. So $P$ is not an ordered chain. Moreover, it can be assumed that $a_{i} \in(x \wedge y, x]$ and $a_{j} \in(x \wedge y, y]$. Then $a=a_{i} \wedge a_{j}=x \wedge y$ and $a<a_{k}$. Thus $a_{k}$ and either $a_{i}$ or $a_{j}$ are comparable which cannot happen. So $\boldsymbol{P}$ is in fact atriodic. Conversely, suppose that $P \subset X$ is atriodic, chainable, and closed. Let $\inf P=a$. Assume that $P$ has three distinct quasi suprema; namely, $a_{1}, a_{2}$, and $a_{3}$. Then $a<a_{1}, a_{2}, a_{3}$. If $a=a_{i} \wedge a_{j}$ for all $i, j \in\{1,2,3\}$, then $\bigcup_{n=1}^{3}\left[a, a_{n}\right]$ would be a triod in $P$ by Lemma 6.6. So assume that $a<a_{1} \wedge a_{2}$. Now $a_{1}$ and $a_{2}$ are not comparable. Consequently, $\left[a_{1} \wedge a_{2}, a\right] \cup\left[a_{1} \wedge a_{2}, a_{1}\right] \cup$ $\left[a_{1} \wedge a_{2}, a_{2}\right]$ is a triod in $P$ by Lemma 6.6. Thus $P$ has at most two quasi suprema. Now apply Lemmas $6.2,6.4$, and 6.5 .

Let $Y$ be a nested space and give $Y$ the semitree structure of $\S 5(\mathrm{~b})$. Then $A \subset Y$ is atriodic if and only if $A$ does not contain what is usually called a simple triod - a union of three nondegenerate arcs with exactly one point common to any two of them.

COROLlary 6.8. Let $A$ be a nondegenerate subset of the nested space $(Y, T)$. Then $A$ is an arc if and only if $A$ is arcwise connected, atriodic, and $T$ closed.

Proof. If $A \subset Y$ is an arc, then $A$ is obviously arcwise connected and 
$T$ closed since $(Y, T)$ is Hausdorff. Further, $A$ is a chain and is atriodic by Theorem 6.7. Conversely, let $A$ be arcwise connected, atriodic, and $T$ closed. Then $A$ is chainable and closed since $T \subset T(P)$. Theorem 6.7 implies that $A$ is a chain and thus is an arc.

As an immediate consequence of Corollary 6.8, a nondegenerate space $A$ is an arc if and only if $A$ is atriodic and nested. This result admits more direct proofs, and it, in fact, is equivalent to Theorem 6.7 and Corollary 6.8. But the above line of argument is perhaps the most illuminating.

7. Topological characterizations of semitrees. Three purely topological characterizations of semitrees are given in this section. The first, in terms of the nested spaces introduced by Young in 1946, amounts to putting together results of $\S \S 4$ and 5. The second, in terms of the mobs of Wallace, generalizes a result of Ward and represents the fruits of another paper. The third and completely new characterization is in terms of box products. The next two theorems can be viewed as extending theorems of Harris [10] to a noncompact, nonmetric setting.

THEOREM 7.1. Let $\left(X, P, \leqslant_{e}\right)$ be a semitree. Suppose that

(i) $T$ is a weakly order compatible Hausdorff topology for $X$;

(ii) every arc in $(X, T)$ is a chain in $P$. Then $(X, T)$ is a nested space. Suppose, in addition, that $T$ is strongly order compatible. Then conditions (i) and (ii) are redundant, and the cutpoint order $\preceq_{e}$ with basepoint $e$ equals $\leqslant_{e}$.

Proof. Only one minor point has not been proved in $\S \S 4$ and 5(b); namely, that $\leqslant_{e} \subset \preceq_{e}$ when $T$ is strongly order compatible. So let $e \neq x<_{e} y$. Then $X \backslash\{x\}=(X \backslash M(x)) \cup(M(x) \backslash\{x\})$ is a separation with $e \in X \backslash M(x)$ and $y \in M(x) \backslash\{x\}$. Thus $x \prec_{e} y$, as required.

THEOREM 7.2. Let $(X, T)$ be a nested space. Then $X$ admits the structure of a semitree $\left(X, P, \leqslant_{e}\right)$ wherein $P$ consists of the arcs in $(X, T), T$ is weakly order compatible, and the cutpoint order $\varsigma_{e}$ with basepoint $e$ is contained in $\leqslant_{e}$. Now $T$ is order compatible at $e$ if and only if $M(x)$ is $T$ closed for all $x \in X$. If $T$ is order compatible at $e$, then $\varsigma_{e}=\leqslant_{e}$ if and only if $T$ is strongly order compatible.

Proof. Again only one thing remains to be proved: if $\preceq_{e}=\varsigma_{e}$, then $M(x) \backslash\{x\}$ is open for each $x \in X$. This is obvious for $x=e$. So let $e \neq x \in X$ and $y \in M(x) \backslash\{x\}$. Then $e \neq x<y$; so write $X \backslash\{x\}=A \cup B$ where $A$ and $B$ are separated, $e \in A$, and $y \in B$. Then $y \in B \subset M(x) \backslash\{x\}$ where $B$ is open, showing that $M(x) \backslash\{x\}$ is open.

Acyclic spaces can be characterized in a similar fashion using Axioms 1-4 and 6. 
The terms used in the following two theorems are defined in [16].

THEOREM 7.3. Suppose that $(X, T)$ is a commutative idempotent monotone mob that satisfies condition 0 . If each maximal totally ordered subset of $X$ is compact, then $X$ admits the structure of a semitree with $T$ strongly order compatible.

A semitree $(X, P, \leqslant)$ is continuous on the diagonal (with respect to a topology $T$ for $X$ ) if and only if for each $x \in X$ and each open set $U$ containing $x$, there exists an open set $V$ containing $x$ such that $V \wedge V=$ $\{y \wedge z \mid y, z \in V\} \subset U$.

THEOREM 7.4. Let $(X, P, \leqslant)$ be a semitree with strongly order compatible topology $T$. If $(X, T)$ is continuous on the diagonal, then $X$ admits the structure of a commutative idempotent monotone mob that satisfies condition 0 and has compact maximal totally ordered subsets.

Theorems 7.3 and 7.4 were proved in [16]. Observe that any tree satisfies the hypotheses of either theorem [25]. The following lemma provides further examples.

LEMmA 7.5. A semitree $(X, P, \leqslant)$ is continuous on the diagonal with respect to any locally chainable topology $T$ for $X$.

Proof. Let $x \in U \in T$. Let $V$ be the chain component of $U$ containing $x$. Then $V$ is $T$ open and $V \wedge V \subset U$.

Until Theorem 7.10, let $\left(X, P, \leqslant_{e}\right)$ be a semitree, let $M$ consist of the $\leqslant_{e}$ maximal elements in $X$, and give $X$ the chain topology $T(P)$. Recall that $X=\bigcup\{[e, m] \mid m \in M\}$ by Theorem 3.1. Consider the product set $B=X\{[e, m] \mid m \in M\}$. For each $m \in M$, give $[e, m]$ the order topology. The box product topology for $B$ has basis consisting of all boxes $\times\left\{U_{m} \mid m \in M\right\}$ where $U_{m}$ is open in $[e, m]$ for each $m \in M$. Motivation for using this topology on $B$ can perhaps be provided by Lemma 4.12 .

For each $m \in M$, define

$$
A_{m}=\{a \in B \mid \text { if } n \in M \text { and } n \neq m \text {, then } a(n)=e\},
$$

and let $A=\left\{A_{m} \mid m \in M\right\}$. The axes in $B$. consist of the union $A=\cup A$. For each $m \in M$, let $\pi_{m}: B \rightarrow[e, m]$ be projection and let $f_{m}$ be $\pi_{m}$ restricted to $A_{m}$. The next lemma is well known.

LEMMA 7.6. For each $m \in M, f_{m}$ is a homeomorphism.

LEMmA 7.7. The subspace topology on $A$ induced by $B$ is exactly $T(A)$, the strong topology in $A$ induced by $A$ where each $A_{m} \in A$ is given the subspace topology induced by $B$. 
Proof. Let $T_{A}$ denote the subspace topology on $A$ induced by $B$. Clearly $T_{A} \subset T(A)$ since $T(A)$ is the largest topology that preserves the given topology on each $A_{m} \in$ A. Let $a \in U \in T(A)$. Suppose first that $a\left(m_{0}\right) \neq e$ for some $m_{0} \in M$. Then $U_{m_{0}}=U \cap A_{m_{0}}$ is open in $A_{m_{0}}$, and $a \in$ $\pi_{m_{0}}^{-1}\left(f_{m_{0}}\left(U_{m_{0}} \backslash\{e\}\right)\right) \cap A \subset U$ showing that $U \in T_{A}$. Second suppose that $a(m)=e$ for all $m \in M$. Then $U_{m}=U \cap A_{m}$ is open in $A_{m}$ for all $m \in M$, and $a \in X\left\{f_{m}\left(U_{m}\right) \mid m \in M\right\} \cap A \subset U$ showing that $U \in T_{A}$. Thus $T_{A}=T(A)$ completing the proof.

Define $f: A \rightarrow X$ by the formula $\left.f\right|_{A_{m}}=f_{m}$ for all $m \in M$. As an easy consequence of Lemma 7.7, the function $f$ is a continuous surjection. A function $g: Y \rightarrow Z$, where $Y$ and $Z$ are topological spaces, is a quotient map if and only if $g$ is a continuous surjection such that $g^{-1}(U)$ open in $Y$ where $U \subset Z$ implies that $U$ is open in $Z$.

LEMMA 7.8. The function $f$ is a quotient map.

Proof. Let $U \subset X$ be such that $f^{-1}(U)$ is open in $A$. Then $f^{-1}(U) \cap$ $A_{m}$ is open in $A_{m}$ for all $m \in M$. So $U \cap[e, m]=f_{m}\left(f^{-1}(U) \cap A_{m}\right)$ is open in $[e, m]$ for all $m \in M$. Finally, $U$ is open in $X$ by Theorem 4.5 and Lemma 4.6.

However, the function $f$ need not be pseudo open (much less closed, open, or perfect). If $X$ is a fan, then $f$ is a homeomorphism. Therefore with the chain topology any fan is homeomorphic to the axes in a box product of arcs. Conversely, $A$ is closed in $B$ and admits the structure of a semitree in a natural way.

Define the usual equivalence relation $K(f)$ in $A$ by the formula $a K(f) a^{\prime}$ if and only if $f(a)=f\left(a^{\prime}\right)$ where $a, a^{\prime} \in A$. Let $p$ be the natural projection onto $A / K(f)$, the decomposition space of $f$. Then there is a commutative diagram

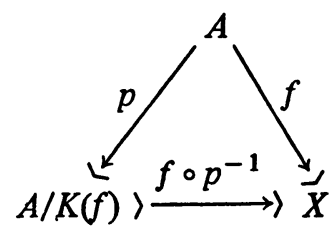

where $f \circ p^{-1}$ is a homeomorphism [7].

THEOREM 7.9. With the chain topology any semitree is homeomorphic to a quotient space of the axes in a box product of arcs.

Simply put, a semitree is a quotient space of a fan. Theorem 7.9 is in one sense the best possible theorem. The topologies in the theorem are $T(A)$ and $T(P)$, and these are the maximal strongly order compatible topologies for $A$ 
and $X$, respectively. If the minimal strongly order compatible topologies, $T_{P}$ and $T_{0}$, are used instead, then the function $f$ need not even be continuous (observe that $T_{P}$ is the product topology restricted to $A$ ). For an example, reflect the comb $\left(C, T_{0}\right)$ of $\S 5(\mathrm{c})$ about the line $x=1$. Then for any $e \in X$ there is $x \in X$ such that $f^{-1}\left(X \backslash M_{e}(x)\right) \notin T_{P}$ where $f$ is the function corresponding to $e$. Moreover, the situation in general cannot be rectified by simply enlarging $T_{P}$. For an example, reflect the broom $B$ about the line $x=1$ [20]. Then for any $e \in X$ there exists $U \in T(P) \backslash T_{0}$ with $f^{-1}(U) \in T_{P}$ where $f$ is the function corresponding to $e$. Thus $f:\left(A, T_{A}\right) \rightarrow\left(X, T_{0}\right)$ is not a quotient map for any choice of strongly order compatible topology $T_{A}$ (that is, $T_{P} \subset T_{A} \subset T(A)$ ). Part of the next theorem is any easy application of Theorem 7.9 or the results of $\S 5(\mathrm{c})$. Further applications of Theorem 7.9 await developments in the theory of box products.

THEOREM 7.10 (NAITO-WolK). For a semitree $(X, P, \preccurlyeq)$, the following are equivalent:

(i) $X$ is finite;

(ii) $X$ contains no infinite totally unordered subset;

(iii) $X$ has a unique weakly order compatible topology;

(iv) $(X, T(P))$ is compact.

Proof. Obviously (i) and (ii) are equivalent and (iii) implies (iv). Also (i) implies (iii) was shown in $\S 5$ (c). For (iv) implies (i), use the open cover $\{X \backslash(M \backslash\{m\}) \mid m \in M\}$. To see that (i) implies (iv) from Theorem 7.9, observe that if $X$ is finite, then the box and product topologies on $B$ coincide and $B$ is compact.

Similarly, a semitree $X$ is countable if and only if $(X, T(P))$ is Lindelö. Also any fan $(X, T(P))$ is seen to be completely normal and paracompact. Thus, if a fan $(X, T(P))$ has real arcs, then $(X, T(P))$ is Lindelö if and only if it is separable. In this connection note that a countable semitree $(X, T(P))$ with real arcs is contractible. These are probably only partial results, and so the proofs are omitted.

The final theorem is quite striking and uses in its proof many ideas of $\S \S 2-5$. It indicates close parallels between semitrees and one dimensional simplicial complexes, and it extends (except for the countability and metrizability statements) to semitrees with arbitrary arcs.

THEOREM 7.11. If $\left(X, P, \leqslant_{e}\right)$ is a semitree with real arcs, then the following properties are equivalent for the space $(X, T(P))$ : (i) compactness; (ii) first countability; (iii) second countability; (iv) metrizability; (v) countable compactness; (vi) $\sigma$ compactness; (vii) local compactness; (viii) $X$ contains no infinite combs or fans; (ix) $X$ is a dendrite. 
Proof. By prior results, (i) implies all of the other statements. It will be shown that (ii) implies (viii) and (viii) implies (i). Similarly, (v) or (vii) imply (viii) which will complete the proof. Suppose that $(X, T(P))$ is first countable. Assume that $X$ contains two infinite sets $\left\{a_{i}\right\}_{i=1}^{\infty}$ and $\left\{b_{i}\right\}_{i=1}^{\infty}$ such that $\left\{a_{i}\right\}$ is totally ordered and if $a_{0}=\sup a_{i}$, then $a_{i}<b_{i}$ and $a_{0} \wedge b_{i}=a_{i}$ for each $i=1,2, \cdots$; that is, assume that $X$ contains an infinite comb. Let $x$ be a cluster point of $\left\{a_{i}\right\}_{i=1}^{\infty}$ in $\left[e, a_{0}\right]$, and let $U_{1}, U_{2}, \cdots$ be a countable open basis at $x$. For each $i=1,2, \cdots$, let $a_{n_{i}} \in U_{i} \backslash\left\{x, a_{n_{1}}, \cdots\right.$, $\left.a_{n_{i-1}}\right\}$, let $U_{i} \cap\left[a_{n_{i}}, b_{n_{i}}\right]=\left[a_{n_{i}}, c_{n_{i}}\right)$, and let $d_{n_{i}} \in\left(a_{n_{i}}, c_{n_{i}}\right)$. Then $X \backslash \bigcup_{i=1}^{\infty}\left[d_{n_{i}}, b_{n_{i}}\right]$ is a $T(P)$ neighborhood of $x$ that contains no $U_{i}$ for $i=1,2, \cdots$. This is a contradiction, and so $X$ contains no infinite combs. Assume that $X$ contains an infinite totally unordered set $\left\{a_{i}\right\}_{i=1}^{\infty}$ and a point $x$ such that $x=a_{i} \wedge a_{j}$ for all $i, j=1,2, \cdots$. Let $U_{1}, U_{2}, \cdots$ be a countable open basis at $x$. For each $i=1,2, \cdots$, let $U_{i} \cap\left[x, a_{i}\right]=\left[x, b_{i}\right)$ and let $c_{i} \in\left(x, b_{i}\right)$. Then $X \backslash \bigcup_{i=1}^{\infty}\left[c_{i}, a_{i}\right]$ is a $T(P)$ neighborhood of $x$ that does not contain $U_{i}$ for any $i=1,2, \cdots$. This is a contradiction, and thus $X$ contains no infinite fans. Therefore, (ii) implies (viii). Suppose now that $X$ contains no infinite combs or fans. Assume that $X$ has infinitely many maximal elements. Consider $B_{1}$, the branches at $e$. If there are infinitely many branches at $e$, then it would be easy to construct an infinite fan at $e$. So there are finitely many branches at $e$, and one of them contains infinitely many maximal elements. Let $B_{1} \in B_{1}$ be such a branch, and let $m_{1} \in B_{1}$ be maximal. Consider the following family of branches:

$B_{2}=\left\{B \mid B\right.$ is a nondegenerate branch at $a \in\left(e, m_{1}\right)$ and $\left.B \cap\left[e, m_{1}\right]=\{a\}\right\}$.

Considering $B_{1}$ as a subsemitree (a chainable closed subset) of $X$ and applying Corollary 3.3 to $B_{1}$, one obtains $B_{1}=\left[e, m_{1}\right] \cup \cup B_{2}$. If $B_{2}$ contains infinitely many branches, then there are two possibilities. On the one hand there might be infinitely many points $a \in\left(e, m_{1}\right)$ having nondegenerate branches $B$ at $a \in\left(e, m_{1}\right)$, and it would be possible to construct an infinite comb in $B_{1}$. On the other hand there might be infinitely many branches at a single point $a \in\left(e, m_{1}\right)$, and an infinite fan could be constructed. So $B_{2}$ contains only finitely many branches, and one of them contains infinitely many maximal elements. Let $B_{2} \in B_{2}$ be such a branch at $a_{1} \in\left(e, m_{1}\right)$, and let $m_{2} \in B_{2}$ be maximal. Proceed inductively with this construction obtaining two infinite sets $\left\{a_{i}\right\}_{i=1}^{\infty}$ and $\left\{m_{i}\right\}_{i=1}^{\infty}$ such that $\left\{a_{i}\right\}$ is totally ordered and if $a_{0}=$ $\sup a_{i}$, then $a_{i}<m_{i}$ and $a_{0} \wedge m_{i}=a_{i}$ for each $i=1,2, \cdots$. Thus an infinite comb has been constructed in $X$, a contradiction. So $X$ in fact has only finitely many maximal elements, and (viii) implies (i). 


\section{REFERENCES}

1. R. F. Arens, On the construction of linear homogeneous continua, Bol. Soc. Mat. Mexicana 2 (1945), 33-36. MR 7, 277.

2. D. P. Bellamy, Composants of Hausdorff indecomposable continua; a mapping approach, Pacific J. Math. 47 (1973), 303-309.

3. K. Borsuk, A theorem on fixed points, Bull. Acad. Polon. Sci. Cl. III 2 (1954), 17-20. MR 16, 275.

4. J. J. Charatonik, On ramification points in the classical sense, Fund. Math. 51 (1962/63), 229-252. MR 26 \#743.

5. J. J. Charatonik and C. A. Eberhart, On smooth dendroids, Fund. Math. 67 (1970), 297-322. MR 43 \#1129.

6. - On contractible dendroids, Colloq. Math. 25 (1972), 89-98, 164.

MR 46 \#8193.

7. J. Dugundji, Topology, Allyn and Bacon, Boston, Mass., 1966. MR 33 \#1824.

8. O. Frink, Jr., Topology in lattices, Trans. Amer. Math. Soc. 51 (1942), 569-582. MR 3, 313.

9. R. W. Hansell, Monotone subnets in partially ordered sets, Proc. Amer. Math. Soc. 18 (1967), 854-858. MR 35 \#6594.

10. J. K. Harris, Order structures for certain acyclic topological spaces, Thesis, University of Oregon, 1962.

11. W. Holsztyńnki, Fixed points of arcwise connected spaces, Fund. Math. 64 (1969), 289-312. MR 40 \#2008.

12. S. Mardešić, A locally connected continuum which contains no proper locally connected subcontinuum, Glasnik Mat. Ser. III 2 (22) (1967), 167-178. MR 36 \#4526.

13. L. K. Mohler, Partial orders and the fixed point property for hereditarily unicoherent continua, Thesis, University of Oregon, 1968.

14. - A fixed point theorem for continua which are hereditarily divisible by points, Fund. Math. 67 (1970), 345-358. MR 41 \#6196.

15. T. B. Muenzenberger and R. E. Smithson, Fixed point structures, Trans. Amer. Math. Soc. 184 (1973), 153-173.

16. - Mobs and semitrees, J. Austral. Math. Soc. (to appear).

17. - Refluent multifunctions on semitrees, Proc. Amer. Math. Soc. 44 (1974), 189-195.

18. T. Naito, On a problem of Wolk in interval topologies, Proc. Amer. Math. Soc. 11 (1960), 156-158. MR 22 \#1528.

19. R. L. Plunkett, A fixed point theorem for continuous multi-valued transformations, Proc. Amer. Math. Soc. 7 (1956), 160-163. MR 19, 301.

20. R. E. Smithson, A note on acyclic continua, Colloq. Math. 19 (1968), 67-71. MR 37 \#2190.

21. - Topologies generated by relations, Bull. Austral. Math. Soc. 1 (1969), 297-306. MR 41 \#2605.

22. L. A. Steen and J. A. Seebach, Jr., Counterexamples in topology, Holt, Rinehart, and Winston, New York, 1970. MR $42 \# 1040$.

23. L. B. Treybig, Concerning homogeneity in totally ordered, connected topological space, Pacific J. Math. 13 (1963), 1417-1421. MR 28 \#2526.

24. L. E. Ward, Jr., A note on dendrites and trees, Proc. Amer. Math. Soc. 5 (1954), 992-994. MR 17, 180. 
25. L. E. Ward, Jr., Mobs, trees, and fixed points, Proc. Amer. Math. Soc. 8 (1975), 789804. MR 20 \#3516.

26. - On dendritic sets, Duke Math. J. 25 (1958), 505-513. MR 20 \#4818.

27. - A fixed point theorem for multi-valued functions, Pacific J. Math. 8 (1958), 921-927. MR 21 \#2215.

28. - A fixed point theorem for chained spaces, Pacific J. Math. 9 (1959), 1273-1278. MR 21 \#7496.

29. - Characterization of the fixed point property for a class of set-valued mappings, Fund. Math. 50 (1961/62), 159-164. MR 24 \#A2956.

30. — Topology, Marcel Dekker, New York, 1972.

31. G. T. Whyburn, Analytic topology, Amer. Math. Soc. Colloq. Publ., vol. 28, Amer. Math Soc., Providence, R. 1., 1942. MR 4, 86.

32. E. S. Wolk, Order-compatible topologies on a partially ordered set, Proc. Amer. Math. Soc. 9 (1958), 524-529. MR 20 \#3079.

33. - On partially ordered sets possessing a unique order-compatible topology, Proc. Amer. Math. Soc. 11 (1960), 487-492. MR 22 \#2568.

34. G. S. Young, Jr., The introduction of local connectivity by change of topology, Amer. J. Math. 68 (1946), 479-494. MR 8, 49.

35. - Fixed point theorems for arcwise connected continua, Proc. Amer. Math. Soc. 11 (1960), 880-884. MR 22 \#8486.

DEPARTMENT OF MATHEMATICS, KANSAS STATE UNIVERSITY, MANHATTAN, KANSAS 66506

DEPARTMENT OF MATHEMATICS, UNIVERSITY OF WYOMING, LARAMIE, WYOMING 82071 